Dear Author,

Please, note that changes made to the HTML content will be added to the article before publication, but are not reflected in this PDF.

Note also that this file should not be used for submitting corrections. 


\title{
Pharmacy workforce to prevent and manage non-communicable diseases in developing nations: The case of Nepal
}

Saval Khanal, B.Pharm., M.Sc. Ph.D. student ${ }^{\mathrm{a}, \mathrm{b}, *}$, Lisa Nissen ${ }^{\mathrm{c}}$, Lennert Veerman ${ }^{\mathrm{d}}$, Samantha Hollingworth ${ }^{\mathrm{a}}$

${ }^{\text {a }}$ School of Pharmacy, The University of Queensland, Australia

${ }^{\mathrm{b}}$ Sankalpa Foundation, Pokhara, Kaski, Nepal

${ }^{\mathrm{c}}$ School of Clinical Sciences, Queensland University of Technology, Australia

${ }^{\mathrm{d}}$ School of Public Health, The University of Queensland, Australia

\begin{abstract}
Summary
Non-communicable diseases (NCDs, e.g. cardiovascular diseases, cancer, chronic respiratory diseases and diabetes mellitus) are main causes of mortality and morbidity in developing countries, including Nepal. Nearly half of the deaths in Nepal are caused by NCDs. Nepal lacks adequate human resources to prevent and manage NCDs, but the skills and expertise of pharmacists in Nepal are underused. There is evidence from many countries that pharmacists can contribute substantially to the prevention and management NCD. We aim to describe the opportunities and challenges for pharmacists to prevent and manage NCDs in Nepal. Pharmacists can contribute by screening and monitoring NCDs; counseling on lifestyle; providing medication therapy management services; promoting public health; and providing other pharmaceutical services. Challenges to the implementation of some of these activities in the current context include inadequate training of pharmacists in NCD prevention and management, the cost of pharmaceutical services to patients and government, and the existing health care service delivery model. There is a need for health services research to determine how pharmacists can be best used to prevent and manage NCDs in Nepal.

(c) 2015 Elsevier Inc. All rights reserved.
\end{abstract}

Keywords: Nepal; Non-communicable diseases; Role of pharmacists

\section{Introduction}

Non-communicable diseases (NCDs) are a major public health problem in Nepal. Two in five $(42 \%)$ of all deaths in Nepal are caused by four common NCDs: cardiovascular diseases; cancer; chronic respiratory diseases; and diabetes mellitus. ${ }^{1}$ Nepal has recently developed an action plan (2014-2020) for the prevention and control of NCDs with a vision - 'all people of Nepal enjoy the highest attainable status of health, well-being and quality of life at every age, free of preventable

* Corresponding author. School of Pharmacy, Pharmacy Australia Centre for Excellence, The University of Queensland, 20 Cornwall Street, Woolloongabba, QLD 4103, Australia.

E-mail address: khanalsaval@gmail.com,s.khanal@uq.edu.au (S. Khanal). 
NCDs, avoidable disability and premature death.' The action plan emphasizes the revitalization and reorientation of health care services for health promotion, disease prevention, early detection and integrated care, particularly at the primary care level. ${ }^{2}$ There is a lack of human resources in health to combat this problem in Nepal. ${ }^{3}$ Pharmacists, by contrast, are underutilized in providing health care services. ${ }^{4}$ This plan provides an opportunity for pharmacists in Nepal to act as one of the major stakeholder to provide primary health care services for NCD patients.

The effective use of pharmacists' knowledge and skill improves health care services delivery. Over time, the role of pharmacists has expanded beyond traditional activities such as compounding, dispensing and packaging medicines. In many countries (especially high-income countries), pharmacists nowadays are more actively involved in activities like ensuring safe and quality use of medicines, promoting public health, providing primary health care services and providing palliative care ${ }^{5-7}$ However in many low-income countries, the role of pharmacists has not been clearly defined and the pharmacy profession has not been well recognized. In Nepal, pharmacists' role in health care provision is not well defined and pharmacy practice is in an emerging phase. ${ }^{4}$ Therefore, this article aims to discuss some ways by which pharmacists can increase their contribution to the health care system, thus describing opportunities and challenges for pharmacists to contribute to prevent and manage NCDs in Nepal.

\section{NCDs in Nepal}

NCDs, especially cardiovascular diseases (CVD), cancer, chronic respiratory diseases and diabetes, are the leading causes of global mortality and morbidity; these diseases kill almost 36 million people globally each year. Nepal, where communicable diseases like childhood diarrhea, pneumonia, and tuberculosis are still important health problems, now faces an additional problem of an escalating burden of NCDs. In $2008,42 \%$ of total deaths were caused by NCDs and this proportion is expected on increase over time. ${ }^{8}$

Q3 The majority of NCD related deaths can be attributed to CVD, which causes $21 \%$ of all deaths in Nepal. Other important causes of NCD deaths are cancer $(7 \%)$, chronic respiratory disease $(5 \%)$, diabetes $(2 \%)$ and other chronic diseases $(7 \%)$. The rise in the number of older people expected to increase the burden of NCDs in Nepal. The proportion of the population aged 65 years or more is expected to rise from $4.2 \%$ in 2000 to $5.8 \%$ in 2025 . Over the period from 2000 to 2030 , a $66 \%$ rise in the number of CVD deaths and a $71 \%$ increase in cancer deaths have been projected from 2000 to 2030 .

Age standardized death rates due to NCDs and Disability Adjusted Life Years (DALYs) rates associated with NCDs show the high burden of NCDs in Nepal. The age standardized death rate due to NCDs was approximately 800 per 100,000 population and age standardized DALY rate due to NCDs were approximately 13,500 per 100,000 population (Table 1). ${ }^{8}$

A high proportion of the Nepalese population is exposed to common risk factors for NCDs. Males and females are exposed about equally to NCD risk factors, apart from much higher use of smokeless tobacco and alcohol consumption among men (Table 2).

\section{Pharmacy workforce in Nepal}

In Nepal, pharmacies can be owned by three different groups - 'pharmacists,' 'assistant pharmacists' and 'professionals. ${ }^{4}$ The law defines a 'pharmacist' as any person with the training and qualification equivalent to Bachelor of Pharmacy, generally known as B.Pharm. (4 years course, done after completion of 12 years of formal schooling); and 'assistant pharmacist' means any person with the qualification equivalent to Diploma in Pharmacy, generally known as D.Pharm. (3 years course, which is done after the completion of 10 years of formal schooling).

'Professionals' are the non-pharmacist retailers with training in dispensing ( $45 \mathrm{~h}$ at beginning was extended to 3 months later), who have the legal right to open a pharmacy and dispense medicines in Nepal. Starting in 1981, professionals were provided training by the Department of Drug

Table 1

Death and DALY of the major NCDs in Nepal (2010)

\begin{tabular}{lll}
\hline NCD & $\begin{array}{l}\text { Death rate (age } \\
\text { conditions } \\
\text { standardized, per } \\
100,000 \text { population) }\end{array}$ & $\begin{array}{l}\text { DALY rate (age } \\
\text { standardized, per } \\
100,000 \text { population) }\end{array}$ \\
\hline CVD & 310 & 2285 \\
Cancers & 120 & 1186 \\
Respiratory & 94.5 & 1102 \\
$\quad$ diseases & & 322 \\
Diabetes & 30.8 & 13,467 \\
All NCDs & 795.9 & \\
\hline
\end{tabular}


Table 2

NCDs risk factors among male and female in Nepal (2011)

\begin{tabular}{lccc}
\hline Risk factors & \% Male & \% Female & Total \% \\
\hline Behavioral risk factors & & & \\
$\quad$ Daily tobacco use & 30.0 & 25.0 & 28.0 \\
Current tobacco use & 36.0 & 29.0 & 32.0 \\
Smokeless tobacco use & 31.2 & 46.0 & 18.6 \\
Less than 5 servings of fruits and vegetables & 61.0 & 64.0 & 62.0 \\
Alcohol consumption & 40.0 & 17.0 & 29.0 \\
Physical inactivity & 12.6 & 15.7 & 14.2 \\
Metabolic risk factors & & & \\
Adult with raised blood pressure & 45.0 & 42.0 & 44.0 \\
Raised blood glucose level & 8.4 & 8.3 & 8.4 \\
Overweight & 9.3 & 8.9 & 9.1 \\
Obesity & 1.3 & 1.6 & 1.4 \\
\hline
\end{tabular}

Administration of the Ministry of Health to manage the shortage of pharmacists in Nepal. With the beginning of formal education in pharmacy, the training for 'Professionals' was stopped in year $1997 .{ }^{3}$

At present there are 15 colleges providing B.Pharm. and 29 colleges providing D.Pharm. education in Nepal. Annually 600 students graduate with B.Pharm. and 1000 with D.Pharm. qualifications in Nepal. Two universities are providing postgraduate courses in pharmacy. Apart from the graduates from Nepal, many Nepalese return to Nepal after completing their education in other countries, particularly India, Bangladesh and Pakistan. ${ }^{4}$

There are approximately 10,000 pharmacies in Nepal. Until recently almost all pharmacies in Nepal were owned and operated by professionals, but currently $10 \%$ of pharmacies are owned and operated by assistant pharmacists. There were only 35 pharmacists working in community pharmacies in $2012 .^{4}$

\section{Current status of pharmacy practice in Nepal}

As mentioned in the previous section, trained providers only run a limited number of pharmacies in Nepal. This may be due to a lack of motivation, inadequate remuneration and a lack of appropriate exposure during their education. Because of the lack of highly skilled manpower in community pharmacies, pharmacy is practiced at a very basic level and is often seen as a business rather than a profession. ${ }^{4}$ However, the recent influx of assistant pharmacists and pharmacists in community pharmacies has led to an expansion of the role of pharmacists. Some of the pharmacists are now providing patient counseling services, regular blood pressure monitoring services, medication record keeping services, medicine information services, first aid treatment and vaccination services. ${ }^{9-12}$

The government drafted National Good Pharmacy Practice Guidelines in 2005, and set standards for pharmacy practice in Nepal. The guidelines have expanded the role of pharmacists in procurement and inventory management in pharmacies; proper storage of medicines; prescription handling; dispensing (filling of prescriptions and preparation of extemporaneous preparations); patient information; patient counseling; medication record and patient follow-up; self-care; health promotion; and pharmacovigilance. ${ }^{7}$ With the increase in number of assistant pharmacists and pharmacists opting for community pharmacy as their work area, and with the development of practice standards, we can expect the pharmacy profession will evolve within some years. A change in paradigm from product-oriented (pharmaceutical industries) to patient-centered (hospital and community pharmacy) can be expected in Nepal.

\section{Potential areas where pharmacists can contribute}

Community pharmacies are the first point of contact for the majority of the Nepalese population to solve their health related problems. ${ }^{12}$ Therefore, they can play vital role in the prevention and management of NCDs. Some of the potential areas where pharmacists can contribute in addition to traditional dispensing are mentioned below.

a) Screening and monitoring NCDs: Pharmacists can make an important contribution to the
279

280 
health care system by screening and monitoring the progression of NCDs in patients. Studies in many countries show that community pharmacists are adequately capable and ideally placed to screen for different conditions including asthma, COPD, diabetes, cardiovascular diseases, breast cancer, bowel cancer, and osteoporosis. ${ }^{6,710,11}$ There is sufficient evidence that pharmacist-operated monitoring programs contribute to the prevention and management of NCDs. Pharmacists have been actively involved in monitoring blood pressure, blood lipid profile, blood glucose level, lung function and anticoagulant therapy. ${ }^{6,7,10,11}$ Replication of these activities by pharmacists in the Nepalese setting can be especially important because of the inadequate number of other health care professionals in the country.

b) Lifestyle counseling: Primary prevention of NCDs is one of the major goals of the World Health Organization Framework for Prevention and Control of NCDs. The major risk factors for NCDs are life style related, like tobacco smoking, harmful use of alcohol, physical inactivity, and inadequate diet. $^{13-15}$ Pharmacists, who are in frequent contact with patients, could potentially provide lifestyle modification education. They are in an ideal position to offer patients information, guidance, and counseling regarding lifestyle changes that can help patients to prevent and manage NCD conditions. Counseling on dietary requirements, encouragement of physical activity, discouraging harmful use of alcohol, and tobacco cessation are a few examples where pharmacists are expected to contribute in Nepal.

c) Medication therapy management: Medication therapy management (MTM) is medical care provided by pharmacists with the aim to optimize drug therapy and improve therapeutic outcomes for patients, often in collaboration with other health care workers. MTM includes five core components: a medication therapy review (MTR), personal medication record (PMR), medication-related action plan (MAP), intervention and/or referral, and documentation and follow-up. ${ }^{16}$ An MTR is a systematic process of collecting patient and medication-related information which occurs during the pharmacist-patient encounter. It is followed by the preparation of a personal medication record for each patient.
From the PMR, pharmacists identify medication-related problems and develop action plans. Pharmacists may interact with patients independently (e.g. adherence counseling and addition of non-prescription medicines) or may refer them to suitable health care professionals (if an intervention is out of the pharmacist's scope of expertise). ${ }^{17-19}$ MTM in case of NCDs can provide patient specific individually-tailored service to patients which may improve the response of patients toward their treatment plan. MTM is practiced in many countries and requires highly trained and skilled pharmacists. The recent shift in pharmacists' interests toward community pharmacy services can help to promote pharmacist-led MTM services in Nepal.

d) Public health and health promotion: The pharmacists being widely accessible and trustworthiness can be one of the good means to promote public health. Pharmacists are regarded as good health educators by the public. There are many models of health promotion by pharmacists (some are even from Nepal). Pharmacist-organized mass education on lifestyle modification, screening of breast cancer, tobacco cessation program and diabetic education program are few examples of health promotion activities conducted by pharmacists. ${ }^{15} \mathrm{~A}$ few pharmacies in Nepal are already providing health promotion services. ${ }^{6}$ Their example can be followed by other pharmacies in Nepal.

e) Other activities: Pharmacists can be involved in various activities in various capacities like health activist to advocate NCD related issues, media person to disseminate NCD related health information, social blogger to write about health information, epidemiologist for NCD surveillance and so on.

\section{Challenges}

The call for pharmacists to step up and recognize their role on prevention and management of NCDs is loud and clear in Nepal. However, there are some barriers to an expanded role for pharmacists. These include the following:

1. Though the number of pharmacists is growing each year; there is still a lack of pharmacists in Nepal.

2. Pharmacists are not adequately trained to provide such services. University curricula 
are still inadequate at training pharmacists on pharmaceutical care, with most universities training pharmacists to work for pharmaceutical industry.

3. The existing pharmacy practice model does not recognize pharmacists as important members of the health care system. Work should be done in policy level to ensure pharmacists has legal rights to provide the primary care to NCD patients, and of course, it will take time to change people's perception toward the services provided by pharmacists.

4. Lack of budget will be another issue. Most health care costs are financed out-of-pocket from patients. Patients may not have money or may not be willing to pay for pharmaceutical services, and therefore suitable financing models have to be found.

\section{Conclusion}

This paper discussed the current situation of pharmacy practice in Nepal, the potential role of pharmacists in responding to the increasing NCD burden, and the challenges facing efforts to promote a greater role of pharmacists in Nepal's health care system. It highlighted that pharmacy practice in Nepal is in emerging phase. NCDs pose a huge threat to Nepal and many other developing nations, and pharmacists can potentially help to prevent and manage NCDs by providing screening and monitoring services; lifestyle modification counseling; medication therapy management, public health and health promotion; and other various services. There must be some studies about how models of pharmaceutical services can be incorporated into existing health care service delivery.

\section{References}

1. Aryal K, et al. Non Communicable Diseases Risk Factors: STEPS Survey Nepal 2013. Kathmandu: Nepal: Health Research Council; 2014.

2. Government of Nepal and World Health Organisation. Multisectoral Action Plan for the Prevention and Control of Non Communicable Diseases (20142020). Kathmandu: Nepal; 2014.

3. Shrestha C, Bhandari R. Insight into human resources for health status in Nepal. Health Prospect 2013;11:40-41.
4. Bhuvan K, Subish P, Izham MM. PharmD education in Nepal: the challenges ahead. Am J Pharm Educ 2011;75. Article 38.

5. Chisholm-Burns MA, et al. US pharmacists' effect as team members on patient care: systematic review and meta-analyses. Med Care 2010;48:923-933.

6. Santschi V, et al. Impact of pharmacist care in the management of cardiovascular disease risk factors: a systematic review and meta-analysis of randomized trials. Arch Intern Med 2011;171: 1441-1453.

7. Ellitt GR, et al. Quality patient care and pharmacists' role in its continuity-a systematic review. Ann Pharmacother 2009;43:677-691.

8. World Health Organization. Global Status Report on Noncommunicable Diseases 2010. World Health Organization; 2011.

9. Bhuvan KC, Alrasheedy AA, Ibrahim MI. Do community pharmacists in Nepal have a role in adverse drug reaction reporting systems? Australas Med J 2013;6:100-103.

10. Mishra $\mathrm{P}$, et al. Medication counseling center in a teaching hospital. JNMA J Nepal Med Assoc 2005;44.

11. Sharma $S$, et al. Impact of community pharmacy-based educational intervention on patients with hypertension in Western Nepal. Australas Med J 2014;7:304.

12. Gyawali S, et al. Pharmacy practice and injection use in community pharmacies in Pokhara city, Western Nepal. BMC Health Serv Res 2014;14:190.

13. Adepu R, Rasheed A, Nagavi B. Effect of patient counseling on quality of life in type- 2 diabetes mellitus patients in two selected South Indian community pharmacies: a study. Indian J Pharm Sci 2007;69:519.

14. Wadden TA, et al. Randomized trial of lifestyle modification and pharmacotherapy for obesity. $N$ Engl J Med 2005;353:2111-2120.

15. Cochran J, Conn VS. Meta-analysis of quality of life outcomes following diabetes self-management training. Diabetes Educ 2008;34:815-823.

16. Bunting BA, Cranor CW. The Asheville Project: long-term clinical, humanistic, and economic outcomes of a community-based medication therapy management program for asthma. J Am Pharm Assoc 2006;46:133.

17. Planas LG, et al. Evaluation of a hypertension medication therapy management program in patients with diabetes. J Am Pharm Assoc 2009;49:164.

18. Christensen DB, et al. Evaluation of a pilot medication therapy management project within the North Carolina state health plan. J Am Pharm Assoc 2006; 47:471-483.

19. Garcia GM, et al. Generating demand for pharmacist-provided medication therapy management: identifying patient-preferred marketing strategies. J Am Pharm Assoc 2008;49:611-616. 